Gdańsk 2020, Nr. 43

https://doi.org/10.26881/sgg.2020.43.03

\author{
Andrzej Kątny \\ Universität Gdańsk / Uniwersytet Gdański \\ https://orcid.org/0000-00025611-8257
}

\title{
Zum Projekt der deutsch-polnischen kontrastiven Grammatik: ein Rückblick
}

\begin{abstract}
In dem Artikel skizziert der Verfasser die Entwicklung der kontrastiven Linguistik in Polen, deren Entwicklung in den USA völlig anders verlief und nach kurzer intensiver Forschung eingestellt wurde. Der Verfasser weist auf die Subdisziplinen hin, in denen die Forschungsergebnisse kontrastiver Linguistik ausgewertet werden können. Im Hauptteil des Artikels wird die Vorgeschichte des von Prof. Ulrich Engel geleiteten Projekts der deutsch-polnischen kontrastiven Grammatik dargestellt und anschließend die Rezeption dieser Grammatik unter polnischen Germanisten besprochen.
\end{abstract}

Schlüsselwörter: kontrastive Linguistik, Deutsch-polnische kontrastive Grammatik und deren Rezeption, Projektleiter Prof. Ulrich Engel

\begin{abstract}
About the project of German and Polish contrastive grammar: a retrospective look. - The article presents the development of contrastive studies in Poland which was different than in the USA, where, after a short period of intensive research, this kind of studies completely disappeared. The author points out the linguistic subdisciplines in which this research can be used. In the main part of the article the author discusses some problems connected with establishing the cooperation between Professor Ulrich Engel from the German Language Institute in Mannheim and Polish Germanists; this cooperation was indispensable for editing a German-Polish contrastive grammar. In the second part, the author presents the reception of the two-volume contrastive grammar, written under the supervision of Professor Engel, among Polish scholars and students of German.
\end{abstract}

Keywords: contrastive linguistics, German-Polish contrastive grammar and its reception, Head of the project - Prof. Ulrich Engel

\section{Einleitung}

Die kontrastive Grammatik entwickelte sich „auf Grund der Bedürfnisse der modernen Theorie des Fremdsprachenunterrichts. Der Aufbau der kontrastiven Grammatik wurde sozusagen von der Spracherlernungspraxis angeregt [...]" (Zabrocki 1970: 37). Zur intensiven Entwicklung der kontrastiven Linguistik $(=\mathrm{KL})$ in Europa ${ }^{1}$ hat die amerikanische Linguistik

1 In Europa gab es kontrastive Ansätze schon viel früher, u. a. in der Prager Schule (V. Mathesius). In Polen kann Adam Kleczkowski (1927) mit seiner, inzwischen in Vergessenheit geratenen, Abhandlung zu Zusammen- 
beigetragen, die das Hauptziel der KL in der Verbesserung der Effektivität des FSU gesehen hat. Hier sind vor allem R. Lado (1957) mit seinem Buch Linguistics across cultures und Ch. Ferguson mit seiner Reihe Contrastive Structures Series zu nennen.

„Die strukturalistischen Initiatoren des Sprachvergleichs hatten ihre Untersuchungen nämlich ursprünglich mit dem Ziel angestellt, auf diese Weise die Probleme zu finden und zu beschreiben, die die Sprecher einer Sprache haben, wenn sie eine andere lernen (vgl. LADO 1964, 215) - in der Annahme, dass die in der Muttersprache ähnlichen Elemente für den Lernenden einfach, die in der Muttersprache verschiedenen Elemente schwierig sein würden. (vgl. LADO 1957: 2)“ (Helbig 1981: 88).

Die kontrastiven Studien sollten helfen, die Lernschwierigkeiten und Fehler vorauszusagen. Als sich nach gewisser Zeit herausstellte, dass sich diese Annahmen nicht erfüllt haben, weil sie eine große Vereinfachung darstellten, kam es zu „Misstrauen, ja zur Skepsis, gegenüber den konfrontativen Untersuchungen“ (Helbig 1981: 89). Deshalb musste „der sogenannte starke, d. h. prognostische Anspruch der kontrastiven Linguistik aufgegeben werden“ (Grucza 1986: 259) und er wurde "gegen Ende der sechziger Jahre zu einer diagnostischen abgeschwächt“ (ebd.), d. h. „sie soll jetzt lediglich helfen, die Ursachen tatsächlich gemachter Fehler zu diagnostizieren" (ebd.).

Die insbesondere für die amerikanische Forschung charakteristischen drei Phasen in der Entwicklung der KL - Überbewertung, pauschale Kritik und im Ergebnis die Unterbewertung der KL - lassen sich in Polen und mehreren Ländern in Mitteleuropa nicht feststellen. In den USA wurden die kontrastiven Projekte Anfang der 1970er Jahre aufgegeben, da sie ihre didaktischen Ziele nicht erfüllt haben. In Polen und einigen europäischen Ländern kam es dagegen zu einem Aufschwung, da man im Prinzip von Anfang an keine illusorischen Anforderungen an diese Subdisziplin stellte; im Klartext: Man hat aus den amerikanischen Erfahrungen und der internationalen Diskussion ${ }^{2}$ eine Lehre gezogen. Grucza (1986: 263) hat Recht, wenn er feststellt,

„dass es sich hier nicht so sehr um eine Krise der kontrastiven Linguistik überhaupt, sondern vorerst lediglich um eine Krise ihrer Voraussetzungen und ihrer Motive handelt, die es verursacht haben, dass ihr Ziele gestellt wurden, die außerhalb des Rahmens ihrer Möglichkeiten liegen.“

Die Ergebnisse der KL können „eine sprachdidaktische Relevanz gewinnen“ - dabei geht es „lediglich um eine erkenntnismäßige Aus- und Verwertung ihrer Forschungsergebnisse“ (ebd.). Die Ergebnisse der KL richten sich an die Verfasser einer pädagogischen Grammatik, Lehrbuchautoren und nicht an die Lerner direkt (vgl. Helbig 1981: 91).

setzungen (1927) als Wegbereiter der kontrastiven Studien gelten. In Frankreich ist hier Charles Bally, Nachfolger von de Saussure auf dem Genfer Lehrstuhl für Allgemeine Sprachwissenschaft, mit seiner Linguistique Générale et Lingustique Française (1932) zu erwähnen. In der Sowjetunion ist L.V. Ščerba mit seiner Phonetik des Französischen (1937) zu nennen (vgl. Sternemann 1977: 5). In vielen praktischen Grammatiklehrwerken des Englischen oder Deutschen des 18. und 19. Jh. lassen sich aus der Lehrpraxis herrührende praktische kontrastive Hinweise finden (vgl. Krzeszowski 1995). Diese Grammatiken und z. T. Lehrwerke stellen die vorwissenschaftliche Phase der KL dar.

2 Vgl. die kritische Zusammenfassung dieser Diskussion u. a. in Grucza (1986), Helbig (1981: 88-101), Rein (1983: 12-27), Tekin (2012: 13-57). 
Für die intensive Entwicklung der KL in Polen (vgl. die Bibliographien von Kątny 1985, 1989) gab es m. E. folgende Gründe:

a) Einführung des Faches „Deutsch-polnische konfrontative Grammatik“ in das Studienprogramm der Germanistik (1974); die Studierenden als zukünftige Deutschlehrer (damals gab es nur diese Spezialisierung) sollten sich die Grundkenntnisse im Bereich der KL aneignen, um diese dann im Unterricht unter Zuhilfenahme didaktischer Prinzipien einzusetzen,

b) Magistranden, Doktoranden sowie andere konnten in ihren Diplom- und Forschungsarbeiten auf ihre Muttersprache zurückgreifen; zugleich war der Zugang zur polonistischen Fachliteratur viel leichter (der Zugang zur westlichen Fachliteratur war bis zur Wende (1989) aus politischen und ökonomischen Gründen stark beschränkt).

Bzdęga (1995: 165) erwähnt noch einen anderen Faktor:

„Die anhaltende Beliebtheit der kontrastiven Problematik ist auf eine von der Muttersprache und vom Stoff her erklärbare Motivation zurückzuführen, u. a. auf thematischen Reichtum an bislang unerforschten Fragen, schließlich auf die glottodidaktischen und translatorischen Zusammenhänge dieser Problematik“.

In den ersten Dekaden wurden hauptsächlich grammatische Strukturen kontrastiv untersucht; fast alle Studien waren unilateral gerichtet, wobei das Deutsche die Ausgangssprache war. Beim unilateralen Vergleich bilden die Strukturen (Formen, lexikalische Einheiten) der Ausgangssprache das Bezugssystem, d. h. das Tertium Comparationis (lat.: 'das Dritte des Vergleichs'; im Weiteren: TC) für die Zielsprache. Methodologisch gesehen verläuft eine unilaterale Analyse in drei Schritten. Im ersten (intralingualen und semasiologischen) Schritt werden die Bedeutungen, verallgemeinerte Funktionen der grammatischen Erscheinungen ermittelt; im zweiten (interlingualen und onomasiologischen) Schritt sucht man nach Äquivalenten ${ }^{3}$ der im ersten Schritt eruierten Sememe. Im dritten (semasiologischen und intralingualen) Schritt können die ermittelten Äquivalente (Formen) auf ihre Bedeutung und Verwendung in der Zielsprache hin untersucht werden:

„Dieser intralinguale Vergleich in der Zielsprache ist darauf gerichtet, die einzelnen sprachlichen Mittel im Hinblick auf ihr Normverhalten, auf spezielle Gebrauchsbedingungen und auf Fragen des Stils gegeneinander abzugrenzen. Der dritte Schritt dient damit der Schaffung der Voraussetzungen für die Vereindeutigung der divergenten Beziehungen von der Ausgangssprache zur Zielsprache." (Hösselbarth 1988: 231)

Beim unilateralen Vergleich werden nur die Strukturen (Bedeutungen) der Ausgangssprache vollständig beschrieben; die Ergebnisse solch eines Verfahrens lassen sich nur in einer Richtung verwenden.

3 „Der zweite Schritt, das interlinguale onomasiologische Vorgehen, entspricht in etwa dem Prozeß, der bei der Translation von Texten im Gehirn des Sprachmittlers abläuft. Diesen Schritt kann man mit einem ,onomasiologischen Tasten' nach den (partiell) bedeutungsgleichen Korrelaten in der Zielsprache vergleichen, wobei das ,Tasten' gestützt wird durch die semasiologische Analyse des ersten Schrittes. [...] Kühlwein / Wills (1981: 9) sprechen beim unilateralen Vergleich von einer ,Sicht der Strukturen einer Sprache durch die 'Brille' einer anderen“" (Hösselbarth 1988: 232). 
Das Problem des TC behandelt viel früher Coseriu (1970: 10); das TC ist nach ihm „das in den Sätzen G e m e in te, d. h. die ,Redebedeutung' der Sätze oder sogar die ,Satzbezeichnung." Er sieht hier eine Verbindung zur Übersetzungspraxis, wo eine ähnliche Frage gestellt wird: „Mit welchen nicht- analogen Sprachmitteln können Sätze der Sprache A und der Sprache B dieselben Sprachinhalte ausdrücken?“ (ebd.). Im Weiteren präzisiert der Forscher die Frage: „Was wird eigentlich in der Sprache B in einer analogen Situation bzw. in bezug auf den gleichen Sachverhalt gesagt?" (ebd.: 12).

Im Bereich der Lexik wandten sich die Forscher hauptsächlich kontrastiven Untersuchungen von Phraseologismen zu; die meisten Analysen waren unilateral. Trotz einer Reihe von Untersuchungen und acht kleineren kontrastiven phraseologischen Lexika fehlt ein großes kontrastives Phraseologisches Wörterbuch Deutsch-Polnisch und Polnisch-Deutsch. Recht gut sind sogenannte „Falsche Freunde des Übersetzers“ bearbeitet worden.

Die pragmatische Wende brachte kontrastive Studien zur Pragmalinguistik hervor. Die Hinwendung der Sprachwissenschaftler zu parole, zu authentischen Sprachsituationen in unterschiedlichen Kommunikationsbereichen, in denen es nicht nur auf grammatische Korrektheit und semantisch-logischen Sinn, sondern auch auf eine angemessene Verhaltensweise ankommt, hat zahlreiche interessante Arbeiten ergeben. Als tertium comparationis fungieren hier im Allgemeinen die einzelnen Sprechakte bzw. die kommunikative Absicht.

Die kontrastive Forschungsperspektive ist (seit längerer Zeit) auch in der Text-, Diskurssowie z. T. in der Medienlinguistik (vgl. dazu Czachur 2011, Olszewska / Kątny/ Socka Hg., 2014; Lüger 2008; Mac 2017: 168-180) präsent.

\section{Kontrastive Linguistik und andere Disziplinen}

Die Kontrastive Linguistik gehört im Allgemeinen zu den komparativen, vergleichenden linguistischen Disziplinen; der vergleichenden Methode bedienen sich außerdem: die historischvergleichende Sprachwissenschaft, die Areallinguistik und die Sprachtypologie. Trotz der Verwendung der gleichen Methode bestehen zwischen ihnen große Unterschiede: Die zwei ersteren sind diachronisch orientiert und auf die Rekonstruktion einer Ursprache oder auf die Rekonstruktion des Ursprungs von Sprachbünden bedacht (vgl. Tekin 2012: 82; Sternemann 1977: 1-4); die Sprachtypologie und die KL sind dagegen synchron und deren Forschungsgegenstand können beliebige Sprachen bilden. In Übereinstimmung mit Zabrocki (1970: 36) betrachte ich die kontrastive ${ }^{4}$ Linguistik als einen Zweig der typologischvergleichenden Sprachwissenschaft.

Die Ergebnisse der KL können u. a. in folgenden Subdisziplinen verwertet werden:

4 Zabrocki (1970) unterscheidet zwischen der konfrontativen und der kontrastiven Linguistik; die erstere soll die Unterschiede und Ähnlichkeiten, die letztere nur Unterschiede zwischen den Sprachen erforschen. In der Linguistik hat sich der zweite Terminus etabliert - er erfasst sowohl Unterschiede als auch Ähnlichkeiten zwischen den untersuchten Sprachen. 
- In der Didaktik des gesteuerten Fremdsprachenunterrichts

Nachdem die in den USA allzu hochgesteckten Ziele und Erwartungen an die KL nicht erreicht worden waren, schwächte man in Europa diese ab, „indem man sie, wie erwähnt, von einer Fehlerprognose auf eine Fehlerdiagnose reduzierte"(Grucza 1986: 260). Im Weiteren betont Grucza (ebd.: 268),

„daß linguistische Feststellungen, Beschreibungen und Theorien von der Glottodidaktik ausgewertet und verwertet werden können und gegebenenfalls von ihr berücksichtigt werden müssen. [...] Es handelt sich aber dabei um keine praktische Anwendung der Linguistik, sondern vorerst lediglich um eine erkenntnismäßige Aus- und Verwertung ihrer Forschungsergebnisse.“

Diese Aus- und Verwertung der Forschungsergebnisse der KL wurde in der Lehrbuchreihe Dein Deutsch, die von F. Grucza (Hg., 1994, 1999) wissenschaftlich betreut wurde, realisiert. In den einzelnen Lehrwerken dieser Reihe wurden entsprechende Übungen und Kommentare sowohl zur Lexik als auch zur Grammatik kontrastiv, unter Berücksichtigung didaktischer Variablen und Ziele angeboten. Im Lehrerhandbuch (Grucza Hg., 1999) wurde eindeutig festgestellt, dass sowohl im grammatischen Stoff als auch in phonetischen Übungen Ergebnisse der KL berücksichtigt wurden. Dies war möglich, da im Autorenteam auch Linguisten vertreten waren.

In den Lehrwerken internationaler Verlage wird das Prinzip der Kontrastivität völlig außer Acht gelassen - wohl aus marktstrategischen Gründen, um an Lerner mit möglichst vielen Muttersprachen heranzukommen und so die Auflagen zu erhöhen. Eventuell kontrastiv orientierte Hinweise kann man in den Lehrerhandbüchern finden, die für jedes Land zum Teil anders und in der jeweiligen Muttersprache der Lerner vorbereitet wurden.

Brdar-Szabo (2001: 201) verweist u. a. auf zwei Erscheinungsformen der Kontrastivität im Unterricht: auf explizite und implizite Bewusstmachung; die letztere „umfasst Strategien, die letztendlich herbeiführen sollen, dass der Lerner durch Inferieren Hypothesen über die Struktur der zu erlernenden Sprache aufstellt und diese ständig verbessert" (ebd.: 202). $\mathrm{Zu}$ diesen Strategien gehören Selektion, Komplexitätsreduktion und Progression. Sie betont, dass „Kontrastivität nur einer unter vielen anderen Faktoren sei, die bei der Steuerung des Fremdsprachenunterrichtes mit berücksichtigt werden sollten" (ebd.: 196). Unterstützung für die Rolle der KL kommt von der Mehrsprachigkeitsdidaktik, „die den Sprachvergleich als ein wichtiges Element des Fremdsprachenlehrens und -lernens in den Fremdsprachenunterricht miteinbezieht" (Tekin 2012: 173). Sie geht von zwei Prämissen aus:

1. „Die Muttersprache wird vom Fremdsprachenlernen nicht ausgeschlossen. Sie bildet vielmehr die Grundlage und den Bezugspunkt für weiteres Sprachenlernen.

2. Die Sprachlernerfahrungen in der ersten Fremdsprache erweitern einerseits den Sprachbestand, der durch die Muttersprache zugrunde gelegt wurde, sie fügen andererseits den Sprachlernerfahrungen, die beim muttersprachlichen Spracherwerb durchlaufen wurden, neue Dimensionen hinzu“" (Neuner 2003: 13).

Dieser Problematik, hauptsächlich aus psycholinguistischer Sicht, ist die Monographie von Chłopek (2011) gewidmet. 
- In der Übersetzungswissenschaft

Ergebnisse der KL spielen noch heute in der Ausbildung von Übersetzern eine wichtige Rolle. Eine Ähnlichkeit zwischen beiden Subdisziplinen sieht man in der Methodologie: In dem 2. (onomasiologischen) Schritt der kontrastiven Analyse geht der Forscher von der Funktion und Bedeutung der gegebenen Struktur aus und fragt nach Entsprechungen in der Zielsprache, wobei er hauptsächlich anhand seines Korpus und/oder seiner Kompetenz nach regulären Äquivalenten sucht.

„Kontrastive Linguistik zielt [...] auf Systemvergleich im Bereich von übereinstimmenden und divergierenden Strukturen; sie operiert auf der Ebene der langue. [...] für kontrastive Analysen von Interesse sind aber in erster Linie Entsprechungen, die der AS-Struktur, so nahe wie möglich kommen" (Koller 2011: 224f.).

Übersetzen ist parole-orientiert. „Übersetzt werden immer Äußerungen und Texte; der Übersetzer stellt Äquivalenz her zwischen AS-Äußerungen und ZS- Äußerungen/Texten, nicht zwischen Strukturen und Sätzen zweier Sprachen“ (ebd.: 224).

Sowohl in der KL als auch in der Translatorik wird dem Begriff der Äquivalenz wichtige Funktion beigemessen. Beim Übersetzen muss jedoch der Übersetzer „die Bedingungen sprachlicher Kommunikation berücksichtigen“ (ebd.: 225). Im Allgemeinen kann ich der Auffassung von Koller (2011:225) zustimmen:

„Die Übersetzungswissenschaft untersucht die Bedingungen von Äquivalenz und beschreibt die Zuordnung von Äußerungen und Texten in zwei Sprachen, für die das Kriterium der Übersetzungsäquivalenz gilt; sie ist Wissenschaft der parole. Die kontrastive Linguistik dagegen untersucht Bedingungen und Voraussetzungen von Korrespondenz (formaler Ähnlichkeit) und beschreibt korrespondierende Strukturen und Sätze; sie ist Wissenschaft der langue."

Aus meiner eigenen Erfahrung kann ich Koller durchaus zustimmen, dass kontrastive Grammatik, Fehler- und Interferenzlinguistik „einen wichtigen Platz" in der Übersetzerausbildung im „Aufbau und Ausbau der fremdsprachlichen Kompetenz “(ebd.: 226) haben.

Mit der pragmatischen Wende sind auch pragmatische kontrastive Studien entstanden sie sind der parole-Ebene zuzuordnen und somit lassen sich engere Verbindungen zu der Translatorik feststellen.

\section{Zur (Vor-)Geschichte des Projekts „Deutsch-polnische kontrastive} Grammatik“(DPG)

Im Folgenden werde ich über die Geschichte des Projekts als dessen aktiver Teilnehmer von Anfang an berichten, indem ich auf schriftliche Aussagen von Prof. Ulrich Engel, auf publizierte Berichte des Instituts für Deutsche Sprache sowie auf andere Texte zur (Vor-)Geschichte des Projekts zurückgreife.

Im März 1969 fand die Jahressitzung des Wissenschaftlichen Rates des IDS zum Thema „Kontrastive Grammatik“ statt. 
„Die Luft war damals sozusagen geschwängert von kontrastiver Linguistik, man erinnert sich: Fergusons Contrastive Structures Series war kurz nach 1960 angelaufen [...], in Budapest schmiedete Juhász die Interferenzproblematik zurecht, an vielen Stellen Europas züngelten kontrastive Projekte auf " (Engel 1999: 361).

„Die Situation war günstig: die Bundesregierung war auf die Stellung der deutschen Sprache in der Welt aufmerksam geworden und hatte Förderungsmaßnahmen ins Auge gefasst. Vor allem auf Empfehlung amerikanischer Germanisten erklärte sich die Kulturabteilung des Auswärtigen Amtes bereit, mehrere kontrastive Projekte des Instituts für deutsche Sprache zu finanzieren“ (Engel 1991: 30).

An dieser Konferenz hat sich u. a. Ludwik Zabrocki (1907-1977) mit dem Referat „Grundfragen der konfrontativen Grammatik“ beteiligt. Einige Monate später schlug er Ulrich Engel während eines Spaziergangs im Kottenforst vor, „gemeinsam eine deutsch-polnische kontrastive Grammatik zu erarbeiten. [...] Ich sagte zu, wir entwickelten sogleich Pläne, einige führende polnische Germanisten wurden zu Mitarbeit gewonnen [...]“(Engel 1999: 361f.). Engel war damals stellvertretender Direktor des IDS und leitete die wissenschaftlichen Arbeiten des Instituts. Zwei Jahre später (1971) unternahm Engel (inzwischen Institutsdirektor) eine Vortragsreise durch Polen, um weitere Gespräche zum geplanten Projekt zu führen.

„In Warschau einigten sich dann J. Czochralski, U. Engel, F. Grucza und L. Zabrocki darauf, eine deutschpolnische Grammatik zu schreiben; die Mitarbeit von Kollegen aus Kraków, Poznań und Wrocław wurde vorgesehen. U. Engel erhielt den Auftrag, einen Arbeitsplan zu entwerfen. Dieser Plan wurde im Herbst 1972 den polnischen Kollegen vorgelegt; er wurde von ihnen ohne Einschränkung akzeptiert" (Engel 1993: 85).

Das Projekt kam aber damals nicht zu Stande, es scheiterte

„an dem politischen Gestrüpp, in das es ungewollt geriet. Erst einmal fand ich in der Kulturabteilung des Auswärtigen Amtes kein Gehör, obwohl die ,Ostverträge damals viele Türen geöffnet hatten. ,Eine deutsch-polnische Grammatik', so wurde mir damals lapidar gesagt, ,wollen wir nicht"' (ebd.).

Das nächste Zitat enthält neben der Faktenlage eine wichtige, von Menschenkenntnis und eigener Erfahrung herrührende, Beurteilung von Prof. Engel (man könnte sagen: aus der Vogelperspektive).

„Das erste Mitarbeiterteam, das sich damals zusammen fand, bestand aus lauter Universitätsprofessoren, die allein schon etabliert waren, also neben ihrem universitären Rang auch schon einen ,Namen besaßen, und die Mehrzahl von ihnen hatte schon eine eigene Schule begründet. Es waren nur drei, aber sie schienen damals ausreichend: Jan Czochralski vom Germanistischen Institut in Warschau, Andrzej

5 Die komplizierte politische Lage und die Einstellung der Abgeordneten zu den Ostverträgen können u. a. die hier erwähnten Quellen beleuchten. „Das Bemühen um die Ratifizierung der Ostverträge war eine harte Auseinandersetzung und ein erbitterter politischer sowie gesellschaftlicher Kampf. Ein Kampf um Sein oder Nicht-Sein der Regierung Brandt, ein Kampf um Sein oder Nicht-Sein der Ostverträge" (Meissner 2015: 310). Da die Ratifizierung der Ostverträge „ernsthaft gefährdet“ war, „entschlossen sich die SPD und FDP, einen kontroversen Kompromiss mit der Opposition zu schließen“ (ebd.: 311). Trotz dieses Kompromisses und der früheren Unterstützung u. a. durch die USA, Großbritannien, Frankreich wurde der Vertrag mit Polen am 17. Mai 1972 mit einer Mehrheit von lediglich einer Stimme durch den Bundestag ratifiziert (ebd.); vgl. dazu auch Jacobsen (1992: 37-40. 
Bzdęga aus Poznań, Franciszek Grucza vom Institut für angewandte Linguistik in Warschau, der auch Koordination in Polen übernehmen sollte. In dieser Konstellation war das Projekt zum Scheitern verurteilt. [...]

Bekannte, angesehene und verdiente Wissenschaftler eignen sich schlecht zur Teamarbeit, es sei denn sie schließen sich aus freien Stücken zusammen“ (Engel 2006: 34).

In dieser Situation wurde der Versuch unternommen, andere Finanzierungsmöglichkeiten zu finden. Der Antrag des IDS auf Finanzierung des Projekts wurde 1976 von der Stiftung Volkswagenwerk gebilligt.

„[...] Deshalb verhandelte U. Engel im Oktober 1976 im Auftrag des Instituts mehrere Tage lang mit F. Grucza als dem Vertreter des Rektors der Universität Warschau, die auf der polnischen Seite federführend sein sollte. Eine Einigung kam dabei, obwohl immer neuen Forderungen der polnischen Seite sukzessiv stattgegeben wurde, nicht zustande. Tieferer Grund für Scheitern der Gespräche war wohl die politische Gesamtsituation. Die Zeit war offensichtlich für eine offizielle (west)deutsch-polnische Zusammenarbeit im kulturellen Bereich noch nicht reif" (Engel 1993: 85-86).

In zwei weiteren Artikeln nimmt Prof. Engel noch einmal zum Fiasko der Verhandlungen Stellung:

„[... aber die einwöchigen Verhandlungen im Herbst 1976 zerschlugen sich - wie ich es heute sehe aus diplomatisch-politischen Gründen. Vor allem hat damals die DDR-Germanistik, die Polen aus verständlichen Gründen als in ihrem Zuständigkeitsbereich liegend ansah, kräftig quergeschossen“ (Engel 1999: 362).

„Unmittelbar vor meiner Abreise nach Polen hatte uns die deutsche Botschaft in einer verschlüsselten Nachricht neue Forderungen der polnischen Seite mitgeteilt: [...]. Dies alles brachte ich als eine Art Geschenk mit, und als in Warschau Tag um Tag neue Wünsche auf den Tisch kamen, konnte ich, mit pauschalen, wenngleich vagen Zusagen meines Ministeriums im Rücken, das Meiste abnicken. [...] Dass diese erhoffte Reaktion nie kam, glaube ich heute zu verstehen. Polen war, trotz der neuen Ostpolitik, weiterhin ein kommunistisch regiertes Land, in dem nicht nur die Sowjetunion, sondern auch die Deutsche Demokratische Republik ihre Interessen zu wahren hatten. Beide werden kräftig gegengearbeitet haben, denn die Bundesrepublik Deutschland war in ihren Augen ein Eindringling, den es in Schranken zu halten galt" (Engel 2006: 35).

Zum Scheitern der Gespräche ${ }^{6}$ nimmt Franciszek Grucza in seiner Monographie (2008: 306) Stellung:

„auch wenn wir anfangs die Zustimmung des Ministeriums erhielten, weitere Schritte in dieser Richtung zu unternehmen, so wurde bald diese Zustimmung rückgängig gemacht, vor allem wegen starken Widerspruchs seitens der damaligen DDR.

6 Ich führe die Feststellung von F. Grucza (2008: 305-306) im vollen Umfang an: „Jeśli chodzi o germanistykę, to wraz z L. Zabrockim (jako przewodniczącym Rady Naukowej ILS) podjęliśmy w warszawskim Instytucie Lingwistyki Stosowanej już w pierwszej połowie lat 70. próbę ukonstytuowania we współpracy z (kierowanym wówczas przez późniejszego profesora Ulricha Engela) Instytutem Języka Niemieckiego w Mannheimie (Institut für deutsche Sprache, Mannheim, RFN) projektu, którego celem miało być opracowanie niemiecko-polskiej i polsko-niemieckiej gramatyki konfrontatywnej, ale choć na początku otrzymaliśmy zgodę władz ministerialnych na podjęcie zmierzających w tym kierunku kroków, to jednak niebawem, przede wszystkim ze względu na ostry sprzeciw ze strony ówczesnej NRD, zgoda ta została cofnięta." 
Prof. Engel gab nicht auf; er leitete mit Erfolg zwei kontrastive Projekte: die deutschserbokroatische und die deutsch-rumänische kontrastive Grammatik. 1988 ist auch seine Deutsche Grammatik erschienen. 1987 und 1988 hielt er Vorträge an einigen Universitäten in Polen und warb „mit Wissen seiner früheren Verhandlungspartner, im Auftrag des Instituts für deutsche Sprache für das deutsch-polnische Projekt“" (Engel 1993: 86). Es gelang ihm (bis 1990) eine Reihe von jüngeren Germanisten und zwei Slawisten für das Projekt zu gewinnen; dank finanzieller Unterstützung der Deutschen Forschungsgemeinschaft (DFG) konnte die Projektarbeit nach dem von Ulrich Engel konzipierten Arbeitsplan zwei Jahre später beginnen. Prof. Engel, der damals nicht mehr am IDS angestellt war, nahm neben der Leitung ${ }^{7}$ den Hauptteil der wissenschaftlichen, redaktionellen und administrativen Arbeit auf sich. Hier muss ich noch erwähnen, dass die Texte am Anfang noch maschinell geschrieben wurden und dass es noch keine elektronische Post gab. Die einzelnen Bearbeitungen wurden von dem Projektleiter und manchmal von den Beratern (Prof. Jan Czochralski und Prof. Krystyna Pisarkowa) gelesen und kommentiert. Zweimal jährlich fanden Arbeitssitzungen statt: im März in Mannheim nach der IDS-Jahreskonferenz und im September meist in Karpacz (Krummhübel im Riesengebirge), seltener in der Grenzstadt Słubice, Wrocław oder Poznań. Im Plenum stellten die Autoren ihre Bearbeitungen vor, nachmittags fanden individuelle Fachgespräche mit dem Projektleiter und zwischen den einzelnen Autoren statt. Die Grammatik ist 1999 im Groos Verlag erschienen, außerdem gab es zwei Nachdrucke in Warschau: im PWN-Verlag sowie Energeia-Verlag.

Die Grundlage für die DPG ${ }^{8}$ bildeten Engels Deutsche Grammatik (1988) sowie seine Syntax der deutschen Gegenwartssprache $\left({ }^{2} 1982,{ }^{3} 1994\right)$; als Grammatikmodell ${ }^{9}$ diente in der Regel (d. h. ohne den Textteil) die dependentielle Verbgrammatik. Das Deutsche war zur damaligen Zeit viel besser beschrieben und erforscht als das Polnische, wo es z. B. kaum Analysen zu den Partikeln und der Valenz gab. Die Forscher mussten also ausgewählte Probleme des Polnischen zuerst erforschen und anschließend einer kontrastiven Analyse unterziehen.

Parallel zu der DPG sollte die Kommunikative Grammatik (= DPK) erarbeitet werden. Dieses Vorhaben wurde in einem Rundbrief an die Projektmitglieder vom 10. März1991 deutlich angesprochen:

\footnotetext{
„Engels Leitungsfunktion impliziert nicht nur die Koordination der Einzelarbeiten, sondern auch die endgültige sprachliche Ausformulierung. Stilistisch hat Engel das Recht, nach Ermessen zu ändern. Soweit es um sachliche Änderungen geht, muß eine Einigung mit dem Autor herbeigeführt werden; scheitert ein solcher Versuch, so muß gegebenenfalls im Plenum diskutiert werden. Engel steht außerdem ständig für die schriftliche oder mündliche Beratung den Mitarbeitern zur Verfügung" (Engel 1990: 3).

8 Außer dem Projektleiter Ulrich Engel gehörten zu den Autoren folgende Mitarbeiter: Lesław Cirko, Antoni Dębski, Alicja Gaca (1931-2006), Alina Jurasz, Andrzej Kątny, Paweł Mecner, Izabela Prokop, Danuta Rytel-Kuc, Roman Sadziński, Christoph Schatte, Czesława Schatte, Eugeniusz Tomiczek (1944-2013) und Daniel Weiss. Als Berater fungierten Jan Czochralski (1924-2004), Krystyna Pisarkowa (1932-2010) und Andrzej de Vincenz (1922-2014).

9 „Engel führt aus, dass für ein kontrastives Unternehmen dieses Umfangs und Anspruchs eine einheitliche theoretische Grundlage erforderlich ist. Diese sieht man - bei der Zustimmung aller Mitarbeiter - in der in Mannheim entwickelten Dependenz-Verb-Grammatik, die im wesentlichen in Engels Deutscher Grammatik (1988) niedergelegt ist. Einige Neuerungen gegenüber dieser Grammatik sind in dem deutsch-rumänischen Projekt des Instituts für Deutsche Sprache realisiert, das demnächst abgeschlossen wird“ (Engel 1990: 2).
} 
„[... in unserer ersten Projektsitzung am 3.12.1990 in Wrocław habe ich kurz über den kommunikativen Teil unserer Grammatik gesprochen und Sie haben auch einen Sonderdruck meines Aufsatzes aus ,Muttersprache' 1990 erhalten."

„[...] Es geht in der ,Kommunikativen Grammatik‘ (fortan: KG) um nichts anderes, als dem Lernenden und auch dem Lehrer sämtliche grammatische Kategorien und Regeln, die für den Spracherwerb benötigt werden, auf eine neue (,kommunikative') Art verfügbar zu machen. Ziel ist also ausschließlich die Vermittlung dessen, was wir als Grammatiker bereits besitzen: der Inhalt der mehr oder weniger herkömmlichen Grammatik“ (Engel 1991a: 1).

Die z. T. parallelen Arbeiten an diesem Vorhaben wurden jedoch nach einigen Jahren aufgegeben, um die Erarbeitung der DPG voranzutreiben. Erst nach dem Erscheinen der DPG wurden die Arbeiten neu aufgenommen. Da einige Mitarbeiter ausschieden und es keine Finanzierung mehr gab, entschied der Projektleiter, die Forschungsergebnisse in einer Reihe von handlichen Bänden zu veröffentlichen. Es erschienen insgesamt fünf kleinere Bände, in denen ausgewählte Bereiche kontrastiv und leserfreundlich besprochen wurden.

Im ersten Band (Engel / Tomiczek 2010) werden die einzelnen partner- und sprecherorientierten Sprechakte und ihre Ausdrucksformen anhand von zahlreichen Beispielen dargestellt. Die weiteren Bände sind betitelt: Argumentieren; Über Sachen Reden; Über Sachverhalte reden. Die in ihnen enthaltenen Abhandlungen und Studien stellen eine gute Grundlage für die in der Zukunft zu bearbeitende kommunikative kontrastive Grammatik dar.

\section{Zur Anwendung und Rezeption der DPG}

Als Benutzer der Grammatik werden folgende Zielgruppen ins Auge gefasst:

„Studierende der jeweils anderen Sprache und ihre Lehrer, also polnische Germanisten und deutsche Polonisten/Slawisten, polnische Deutschlerner und deutsche Polnischlerner in weiterführenden Schulen" (DPG I: 7).

Der Benutzerkreis wurde m. E. zu weit gefasst. Polnische Deutschlerner in Schulen würden zur DPG oder anderen umfangreichen Grammatiken des Deutschen in deutscher Sprache nicht greifen, zumal Sprachkenntnisse der Schüler unzureichend sind und/oder diese Werke in den Schulbibliotheken nicht vorhanden sind. Ambitionierte Lerner können u. a. Mata gramatyka języka niemieckiego. Der kleine Duden ${ }^{10}$ (von R. und U. Hoberg), Gramatyka jezzyka niemieckiego von F. Muller (übersetzt aus dem Französischen) oder unterschiedliche von polnischen Germanisten verfasste Grammatiken ${ }^{11}$ verwenden.

10 Die polnische Übersetzung (von R. und G. Lewicki) enthält kurze Hinweise zu den Unterschieden zwischen den Strukturen beider Sprachen. Im Anhang findet der Lerner ein deutsch-polnisches und ein polnischdeutsches Verzeichnis grammatischer Termini.

11 Z. B.: Czochralski, Jan (1990): Gramatyka niemiecka dla Polaków. Warszawa: Wiedza Powszechna; Łuszczyk, Stanisław / Szulc, Aleksander / Wawrzyniak, Zdzisław (1981): Gramatyka języka niemieckiego. Warszawa: WSiP; Tęcza, Zygmunt (2010): Gramatyka angielska i niemiecka w opisie równolegtym. Rzeszów: Wyd. Uniwersytetu Rzeszowskiego. 
Bei Germanisten ${ }^{12}$ (= Germanistikstudierenden) ist das Bild differenziert. Im ersten Dezennium nach Erscheinen der DPG konnte ich noch Studierende des 2. und 3. Jahres zur Benutzung dieser Grammatik als Begleitlektüre bewegen; in den letzten Jahren ist dies kaum mehr möglich, weil die Studenten über geringere Deutschkenntnisse verfügen (Stichwort: Englisch als erste Fremdsprache im polnischen Schulsystem) und durch intensive Nutzung digitaler Medien kürzere und einfachere Texte (insbesondere in ihrer Muttersprache) vorziehen. Als Lernhilfen zum Fach „Beschreibende Grammatik“ oder „Kontrastive Grammatik Deutsch-Polnisch“ werden lieber Kurzgrammatiken (z. B. Engel 2002; Helbig 1993) und Übungsgrammatiken herangezogen.

Der Wert der DPG besteht in der kontrastiven Darstellung der grammatischen Erscheinungen sowie in der Darstellung des Polnischen in deutscher Sprache und somit für deutsche Muttersprachler. Diese Darstellung ist nach Cirko (2008: 11) „eine Projektion der Deutschen Grammatik Engels aufs Polnische“:

„Die Beschreibung des Polnischen liefert zwar stellenweise interessante Einblicke, sie ist aber keine Grammatik des Polnischen. Der Grund dafür ist die Projektion des Deutschen auf das Polnische, die zur Folge hat, dass viel terminologisch/kategorial/funktional Fremdes in die Beschreibung des Polnischen implementiert wurde“ (Cirko 2008: 9).

\section{Hierzu nimmt Engel (2008: 16) folgendermaßen Stellung:}

„Wenn die Beschreibung für eine Sprache schon im Detail vorliegt, eine einheitliche und theoretisch geschlossene Beschreibung, für die andere jedoch noch nicht, dann ist die Versuchung groß, das, was man auf einer Seite vorfindet, als Folie für die andere Seite zu verwenden und diese dann als Zielsprache zu deklarieren.

[...] Wir haben am Ende vorgelegt, was seinerzeit möglich war, und waren uns schon damals vieler Unzulänglichkeiten bewusst. Eine Revision des Ganzen ist nötig.“

Ein weiterer Einwand von Cirko (2008: 13), der hier die Meinung der Studierenden der Universität Wrocław wiederholt, betrifft die Zerstreuung des Stoffes; er fragt auch danach, „ob alle Distinktionen und Einteilungen wirklich aus der Perspektive der künftigen Philologen in gleichem Maße wichtig sind."

Ulrich Engel sieht den mangelnden Erfolg der DPG in der Tradition an Schulen und Universitäten in Polen. Polen und andere Länder in Osteuropa waren noch mehrere Jahre nach dem Krieg ,im Hinblick auf die Lehrmittel und Begleitmaterialien ein ausgesprochenes Ödland“ (Engel 2008: 18). Die Grammatik von Helbig / Buscha „war ausführlich, gab eine verständliche Darlegung des Stoffes“ (ebd.), sie „hatte eine Reihe moderner Erkenntnisse der Linguistik eingearbeitet" (ebd.) und hat schnell den Markt erobert. Daher gab es im Jahr 2006 folgenden Stand der Dinge:

„Alle, die den Deutschunterricht in Polen prägen - Professoren, Assistenten, Lehrer -, haben ihr Deutsch mit Helbig/Buscha erworben und tragen folglich das Gelernte weiter. Die $d p g$ hat [...] eine völlig andere theoretische Grundlage. Gegen die weiterhin geltende Tradition in Polen kommen wir nicht an“ (ebd.: 19).

12 Meine Einschätzung stützt sich auf meine Lehrpraxis an den Universitäten Poznań und Gdańsk. Zum Teil stütze ich mich auf Erfahrungsaustausch mit Fachkollegen von anderen Universitäten. 
In der Tat hat die DPG in der Grammatik von Helbig und Buscha die stärkste Konkurrenz. Dies ist u. a. auf folgende Faktoren zurückzuführen:

- Die Helbig/Buscha-Grammatik ist seit 1970 in Polen in mehreren Auflagen (zum erschwinglichen Preis) zugänglich; viele Germanisten besitzen das in der DDR herausgegebene Lehrbuch in ihrer Hausbibliothek; in jeder Uni- und Hochschulbibliothek sind mehrere Exemplare vorhanden, die der Leser ausleihen kann.

- Sie wurde für den Deutschunterricht für Ausländer ${ }^{13}$ konzipiert und zwei am Herder-Institut in Leipzig didaktisch aufbereitete Übungsbücher dienen zur Erklärung und Einübung ausgewählter Probleme; außerdem gibt es eine Reihe von Heften zur Didaktisierung ${ }^{14}$ des grammatischen Stoffes - „Zur Theorie und Praxis des Deutschunterrichts für Ausländer“.

Zusammenfassend kann man feststellen, dass die DPG als Lehrbuch zum Grammatikunterricht nur beschränkt von Germanistikstudenten verwendet wird. Sie spielt aber eine wichtige Rolle als brauchbare wissenschaftliche Quelle in Diplomarbeiten, in Doktor- und Habilschriften sowie anderen wissenschaftlichen Studien - insbesondere dann, wenn linguistische, textgrammatische, kontrastive und übersetzungswissenschaftliche Themen bearbeitet werden sollen. Sie ist auch eine wichtige wissenschaftliche Quelle für Typologen sowie deutschsprachige Polonisten und Slawisten.

Zurzeit arbeiten einige der ehemaligen Verfasser an der revidierten Neuauflage der DPG, wobei eine stärkere Lernerzentriertheit im Fokus steht:

„Die Autoren der zweiten Auflage lassen sich von dem Grundgedanken leiten, dass die Form der Darbietung grammatischer Fakten durch Bedürfnisse der Sprachvermittler bestimmt werden muss. Was nur theoretisch vernünftig, aber schwer vermittelbar ist, muss für eine Grammatik, die vor allem dem Fremdsprachenunterricht dienen und in Sprachlehrbücher eingehen soll, entsprechend umgearbeitet werden“" (Rytel-Schwarz u. a. 2012: 14).

Zwei Bände sind bereits erschienen, zwei weitere sind in Bearbeitung. Die Autoren versprechen, die Mängel und Unzulänglichkeiten der ersten Auflage zu beseitigen und neuere Forschungsergebnisse der Polonistik einzubeziehen.

\section{Quellen}

Engel, Ulrich (1990): Protokoll der [1.] Sitzung der Arbeitsgruppe „Deutsch-polnische kontrastive Grammatik" am 2. und 3.12.1990 in Wrocław (Privatbesitz).

Engel, Ulrich (1991): Kontrastive Grammatiken. In: IDS 25, 29-34.

13 „Die Grammatik für den Muttersprachenunterricht kann von der Kompetenz ausgehen, eine Grammatik für den Fremdsprachenunterricht dient (als ein neben anderen auf Kenntnisvermittlung gerichtetes Mittel) dazu, diese Kompetenz aufzubauen“" (Helbig/Buscha 2001: 17).

14 Von der Arbeitsstelle für wissenschaftliche Didaktik am Goetheinstitut wurden im Rahmen des Projekts „Lernschwierigkeiten im Fach ,Deutsch als Fremdsprache“ zu der damaligen Zeit in Polen nicht zugänglich. 
Engel, Ulrich (1991a): Rundbrief an die Projektmitglieder vom 10.3.1991 (Privatbesitz). Engel, Ulrich (2014): Das Institut für deutsche Sprache 1965-1976. In: IDS 50, 64-79.

Grucza, Franciszek (Hg.) (1994-1999): Dein Deutsch (für die V., VI., VII., VIII. Klasse Grundschule; für die I., II., III., IV. Klasse Oberschule [vor der Bildungsreform 1999. Warszawa: Graf-Punkt.

Grucza, Franciszek (Hg.) (1999): Dein Deutsch (für die IV., V., VI. Klasse Grundschule; für die I., II., III. Klasse Oberschule [nach der Bildungsreform 1999]). Warszawa: Graf-Punkt.

Grucza, Franciszek (Hg.) (1994): Poradnik metodyczny do podręcznika do nauki języka niemieckiego w szkotach podstawowych. Klasa V. Warszawa: Graf-Punkt [weitere Lehrerhandbücher 1999, 2000].

IDS 25 - (Hg.) (1989, ${ }^{2}$ 1991): Institut für deutsche Sprache. 25 Jahre. Mannheim: Institut für deutsche Sprache.

IDS 50 - (Hg.) (2014): Ansichten und Einsichten. 50 Jahre Instituts für Deutsche Sprache. Mannheim: Institut für Deutsche Sprache.

\section{Literatur}

Bingen, Dieter (2000): Die Polenpolitik von Willy Brandt und Helmut Kohl. Zwei Ären - zwei Stile. In: Karaskiewicz, Katarzyna (Hg.): Polska - Niemcy - Europa [Polen - Deutschland - Europa]. Warszawa: Oficyna Wydawnicza Rytm, 63-98.

Brdar-Szabó, Rita (2001): Kontrastivität in der Grammatik. In: Helbig, Gerhard u. a. (Hg.), 195-204. Bzdęga, Andrzej (1995): Germanistische Sprachwissenschaft in Polen nach 1945. In: König, Christoph (Hg.): Germanistik in Mittel- und Osteuropa. Berlin, New York: de Gruyter, 160-167.

Chłopek, Zofia (2011): Nabywanie języków trzecich i kolejnych oraz wielojęzyczność. Wrocław: Wydawnictwo Uniwersytetu Wrocławskiego.

Cirko, Lesław (2008): Wissenschaftlichkeit und Anwendbarkeit auf Kollisionskurs? Warum setzt sich die dpg als akademisches Lehrwerk nicht durch? In: Cirko, Lesław / Grimberg, Martin (Hg.), 5-14.

Cirko, Lesław / Grimberg, Martin (Hg.) (2008): Zwischen Lob und Kritik: sechs Jahre Erfahrung mit der Deutsch-polnischen Grammatik ( $1 p g)$.Wrocław / Dresden: Atut / Neisse Verlag.

Cirko, Lesław (2009): Deutsch-polnische kontrastive Grammatik: ihre Rezeption und Neufassung. In: Eichinger, Ludwig M. u. a. (Hg.): Dependenz, Valenz und mehr. Beiträge zum 80. Geburtstag von Ulrich Engel. Tübingen: Groos, 35-43.

Coseriu, Eugenio (1970): Über Leistung und Grenzen der kontrastiven Grammatik. In: Moser, Hugo (Hg.), 9-30.

Czachur, Waldemar (2011): Diskursive Weltbilder im Kontrast. Wrocław: Atut.

DPG - Engel, Ulrich u. a. (1999 / 2000): Deutsch-polnische kontrastive Grammatik. Bd. I, II. Heidelberg: Groos / Warszawa: PWN.

Engel, Ulrich (1988): Deutsche Grammatik. Heidelberg: Groos.

Engel, Ulrich (1993): Deutsch und Polnisch in Kontrast. Bericht über ein Forschungsprojekt. In: Deutsch-Polnisches Jabrbuch der Germanistik 1993. Bonn: DAAD, 71-87.

Engel, Ulrich (1999): Eine neue deutsch-polnische kontrastive Grammatik. Bericht über das Forschungsunternehmen „dpg“. In: Bańczerowski, Jerzy / Zgółka, Tadeusz (Hg.): Linguam amicabilem facere. Ludovico Zabrocki in memoriam. Poznań: Wyd. Naukowe UAM, 361-374.

Engel, Ulrich (2001): dpg - was noch zu tun ist. In: Grucza, Franciszek (Hg.): Tausend Jahrepolnischdeutsche Beziehungen. Sprache - Literatur - Kultur - Politik. Warszawa: Graf-Punkt, 402-410. 
Engel, Ulrich (2002): Kurze Grammatik der deutschen Sprache. München: Iudicium.

Engel, Ulrich (2006): Der Weg nach Polen. Bericht über die kontrastive Grammatik und anders. In: Simmler, Franz / Tomiczek, Eugeniusz (Hg.): Germanistischer Brückenschlag im deutsch-polnischen Dialog. Bd. 1: Sprachwissenschaft. Wrocław / Dresden: Neisse Verlag, 23-40.

Engel, Ulrich (2009): Löcher in der Mauer. In: Convivium. Germanistisches Jahrbuch Polen. Bonn: DAAD, 31-41.

Engel, Ulrich (2019): Lebenslauf bis 2019. In: Studia Germanica Gedanensia 41, 193-202.

Engel, Ulrich / Tomiczek, Eugeniusz (2010): Wie wir reden. Sprechen im deutsch-polnischen Kontrast. Wrocław: Atut, Dresden: Neisse Verlag.

Grucza, Franciszek (1986): Kontrastive Linguistik - Angewandte Linguistik - Glottodidaktik. Bemerkungen zu ihren gegenseitigen Beziehungen. In: Kwartalnik Neofilologiczny XXXIII/3, 257-270.

Grucza, Franciszek (2008): Lingwistyka stosowana. Historia - Zadania - Osiagnięcia. [Angewandte Linguistik. Geschichte, Aufgaben, Errungenschaften]. Warszawa: Euro-Edukacja.

Helbig, Gerhard (1981): Sprachwissenschaft, Konfrontation, Fremdsprachenunterricht. Leipzig: Verlag Enzyklopädie.

Helbig, Gerhard ('1993): Deutsche Grammatik. Grundfragen und Abriß. München: Iudicium.

Helbig, Gerhard / Buscha, Joachim ( ${ }^{1} 1970,{ }^{17} 1996$; 2001): Deutsche Grammatik für den Ausländerunterricht. Leipzig: Enzyklopädie; Berlin: Langenscheidt.

Helbig, Gerhard u. a. (Hg.) (2001): Deutsch als Fremdsprache. Ein internationales Handbuch. 1. Halbband. Berlin / New York: de Gruyter.

Hösselbarth, Lutz (1988): Das unilaterale Vergleichsverfahren in Theorie und Praxis. In: Zeitschrift für Phonetik, Sprachwissenschaft und Kommunikationsforschung 41/2, 230-236.

Jacobsen, Hans-Adolf (1992): Bundesrepublik Deutschland - Polen. Aspekte ihrer Beziehungen. In: Jacobsen, Hans-Adolf / Tomala, Mieczysław (Hg.): Bonn - Warschau 1945-1991. Die deutschpolnischen Beziehungen. Analyse und Dokumentation. Köln: Verlag Wissenschaft \& Politik.

Kątny, Andrzej (1985): Bibliography of German-Polish contrastive studies. In: Papers and Studies in Contrastive Linguistics 20, 141-167.

Kątny, Andrzej (1989): Bibliographie zum deutsch-polnischen Sprachvergleich. Teil III. Stand: März 1989. In: Kątny, Andrzej (Hg.): Studien zur kontrastiven Linguistik und literarischen Übersetzung. Frankfurt/M.: Lang, 65-84.

Kątny, Andrzej (2001): Kontrastive Analysen Deutsch-Polnisch: eine Übersicht. In: Helbig, Gerhard u. a. (Hg.): Deutsch als Fremdsprache. Ein internationales Handbuch. Berlin, New York: de Gruyter, 392-394.

Kątny, Andrzej (2004): Zum Forschungsstand im Bereich der deutsch-polnischen kontrastiven Linguistik. In: Orbis Linguarum 25, 199-212.

Kątny, Andrzej / Schatte, Christoph (1999): Gespräch mit Professor Ulrich Engel. In: Kątny, Andrzej / Schatte, Christoph (Hg.): Das Deutsche von innen und außen. Ulrich Engel zum 70. Geburtstag. Poznań: Wydawnictwo Naukowe UAM, VII-XI.

Kleczkowski, Adam (1927): Złożenia nominalne w języku niemieckim a polskim [Nominale Zusammensetzungen im Deutschen und Polnischen]. In: Prace Filologiczne XII, 522-540.

Koller, Werner (82011): Einführung in die Übersetzungswissenschaft. Tübingen und Basel: A. Francke. Krzeszowski, Tomasz P. (1995): Early Contrastive Studies in England. Gdańsk: Wydawnictwo Uniwersytetu Gdańskiego. 
Kühlwein, Wolfgang / Wilss, Wolfram (1981): Kontrastive Linguistik und Übersetzungswissenschaft Einleitung. In: Kühlwein, Wolfgang u. a. (Hg.): Kontrastive Linguistik und Übersetzungswissenschaft. München: Fink, 6-17.

Lobin, Henning (2018): Bemerkungen zur andauernden Aktualität des Werks von Ulrich Engel. In: Kątny, Andrzej (Hg.): Valenz und Dependenz. Theorie und Praxis. Festschrift für Professor Ulrich Engel zum 90. Geburtstag (= Studia Germanica Gedanensia 39). Gdańsk: Wydawnictwo Uniwersytetu Gdańskiego, 13-19.

Lüger, Heinz-Helmut / Lenk, Hartmut (2008): Kontrastive Medienlinguistik. Ansätze, Ziele, Analysen. In: Lüger, Heinz-Helmut / Lenk, Hartmut (Hg.): Kontrastive Medienlinguistik. Landau: Empirische Pädagogik, 11-28.

Mac, Agnieszka (2017): Textdesign und Bedeutungskonstitution im multimodalen Fernsehtext. Frankfurt/M.: Lang.

Meissner, Lucjan (2015): Willy Brandts Versöhnungspolitik gegenüber Polen 1970-1972. Ein Erinnerungsbeitrag. In: Zeitschrift des Verbandes Polnischer Germanisten 4, 309-318.

Meliss, Meike (2019): Und was kommt danach? Kontrastive Projekte und linguistische Pilgerreisen: Ein persönlicher Blick auf fast drei Dekaden Forschung von Ulrich Engel. In: Studia Germanica Gedanensia 41, 203-214.

Nagórko, Alicja (2000): Rezension zu: Ulrich Engel et al. „Deutsch-polnische Grammatik“ Bd. 1-2. Heidelberg: J. Groos Verlag 1999 / Warszawa: Wyd. Naukowe PWN 2000. In: Biuletyn Polskiego Towarzystwa Jezykoznawczego LVI, 153-158.

Moser, Hugo (Hg.) (1970): Probleme der kontrastiven Grammatik. IDS-Jahrbuch 1969. Düsseldorf: Schwann.

Neuner, Gerhard (2003): Mehrsprachigkeitskonzept und Tertiärsprachendidaktik. In: Hufeisen, Britta / Neuner, Gerhard (Hg.): Mehrsprachigkeitskonzept-Tertiärsprachenlernen - Deutsch nach Englisch. Strasbourg: Council of Europe, 13-34.

Olszewska, Danuta / Kątny, Andrzej \& Socka, Anna (Hg.): Kontrastive Linguistik und interkulturelle Kommunikation (= Studia Germanica Gedanensia 31). Gdańsk: Wydawnictwo Uniwersytetu Gdańskiego.

Rein, Kurt (1983): Einführung in die kontrastive Linguistik. Darmstadt: Wissenschaftliche Buchgesellschaft.

Rytel-Schwarz, Danuta u. a. (2012): Deutsch-polnische kontrastive Grammatik. Bd. 4: Die unflektierbaren Wörter. Zweite, neubearbeitete und ergänzte Auflage. Hildesheim: Olms.

Sternemann, Reinhard u. a. (1977): Einfübrung in die konfrontative Linguistik. 2. Fassung. Berlin: Humboldt-Universität.

Tekin, Özlem (2012): Grundlagen der Kontrastiven Linguistik in Theorie und Praxis. Tübingen: Stauffenburg.

Zabrocki, Ludwik (1970): Grundfragen der konfrontativen Grammatik. In: Moser, Hugo (Hg.), 31-52. 